\title{
A METRICAL RESULT ON THE APPROXIMATION BY CONTINUED FRACTIONS
}

\author{
H. JAGER
}

Abstract. Let $x$ be a real irrational number and $p_{n} / q_{n}, n=0,1,2, \ldots$ its sequence of continued fraction convergents. Define $\theta_{n}=q_{n}\left|q_{n} x-p_{n}\right|$. For almost all $x$ the distribution function of the sequence $\left|\theta_{n+1}-\theta_{n-1}\right|, n=$ $0,1,2, \ldots$ is determined and its expectation calculated.

\section{INTRODUCTION}

Let $x$ be a real irrational number with continued fraction expansion

$$
x=\left[a_{0} ; a_{1}, a_{2}, \ldots\right]
$$

and $p_{n} / q_{n}, n=0,1,2, \ldots$, the corresponding sequence of convergents.

The sequence $\theta_{n}, n=0,1,2, \ldots$, of approximation coefficients of $x$ is defined by

$$
\theta_{n}=q_{n}^{2}\left|x-\frac{p_{n}}{q_{n}}\right|, n=0,1,2, \ldots
$$

One of the most important aspects of the approximation by continued fractions is the fact that this is always a sequence in the unit interval.

The sequence has been extensively studied; see for instance the book [1].

In this paper we determine for almost all irrational $x$ the distribution function of the sequence

$$
\theta_{n+1}-\theta_{n-1}, n=1,2, \ldots,
$$

and as an application, calculate

$$
\lim _{N \rightarrow \infty} \frac{1}{N} \sum_{n=1}^{N}\left|\theta_{n+1}-\theta_{n-1}\right|,
$$

for almost all $x$.

\section{SYMmETRY}

We omit in our notations the dependence on $x$. Define $t_{n}$ and $v_{n}$ for $n=1,2, \ldots$ by

$$
\begin{aligned}
t_{n} & =\left[0 ; a_{n+1}, a_{n+2}, \ldots\right] \\
v_{n} & =\left[0 ; a_{n}, a_{n-1}, \ldots, a_{1}\right] .
\end{aligned}
$$

One has the fundamental equalities

$$
\theta_{n-1}=\frac{v_{n}}{1+t_{n} v_{n}}, \quad \theta_{n}=\frac{t_{n}}{1+t_{n} v_{n}} ;
$$

Received by the editor May 19, 2011.

2010 Mathematics Subject Classification. Primary 11K50.

Key words and phrases. Continued fractions, approximation coefficients, metrical theory. 
see [1, Exercise 5.1.11. They occur already in Koksma's book 2 from 1937, page 25 .

The next theorem lies at the basis of what follows.

Theorem 1. The two-dimensional sequence $\left(t_{n}, v_{n}\right), n=1,2, \ldots$, is for almost all irrational $x$ distributed over the unit square in the $(t, v)$-plane according to the density function $\mu$ where

$$
\mu(t, v)=\frac{1}{\log 2} \frac{1}{(1+t v)^{2}} .
$$

For a proof see 1, Lemma 5.3.11.

In [3] it was shown that the sequence $\left|\theta_{n}-\theta_{n-1}\right|, n=1,2, \ldots$, is distributed over the unit interval with the density function $f$, where

$$
f(z)=\frac{1}{\log 2}\left(\frac{\pi}{2}-2 \arctan z\right)
$$

and as a consequence that

$$
\lim _{N \rightarrow \infty} \frac{1}{N} \sum_{n=1}^{N}\left|\theta_{n}-\theta_{n-1}\right|=\frac{4-\pi}{4 \log 2}=0.30960 \cdots,
$$

for almost all $x$.

From the symmetry in $t$ and $v$ of the two equalities of (1) and of (2) it follows that the density function of the sequence

$$
\theta_{n}-\theta_{n-1}, n=1,2, \ldots,
$$

is an even function on $[-1,1]$. Its explicit form follows at once from (3).

In the sequel, $a$ will always denote a positive integer. Let $R_{a}$ be that part of the unit square in the $(t, v)$-plane where $\frac{1}{a+1} \leq t \leq \frac{1}{a}$. Then $\left(t_{n}, v_{n}\right) \in R_{a}$ if and only if $a_{n+1}=a$.

Now consider the subsequence of (4) by imposing the condition $a_{n+1}=a$ on its terms.

The function

$$
\frac{t-v}{1+t v}
$$

takes its maximum and minimum on $R_{a}$ in, respectively, the lower right-hand and upper left-hand vertex. The maximum is $\frac{1}{a}$, the minimum $-\frac{a}{a+2}$. Hence

$$
\theta_{n}-\theta_{n-1} \in\left[-\frac{a_{n+1}}{a_{n+1}+2}, \frac{1}{a_{n+1}}\right],
$$

from which it follows at once that the subsequence cannot have an even density function because its supporting interval is not symmetric around the origin, except when $a=2$. This case has to be investigated separately.

In this paper we study the same questions for the sequence

$$
\theta_{n+1}-\theta_{n-1}, n=1,2, \ldots,
$$

also a sequence in $[-1,1]$. Instead of (1) we now need

$$
\theta_{n-1}=\frac{v_{n}}{1+t_{n} v_{n}}, \quad \theta_{n+1}=\frac{\left(1-a_{n+1} t_{n}\right)\left(a_{n+1}+v_{n}\right)}{1+t_{n} v_{n}} ;
$$

see [1, Proposition 5.3.6. 
This suggests the map $\Psi_{a}$ from $R_{a}$ onto the $(\alpha, \beta)$-plane given by

$$
\Psi_{a}(t, v)=\left(\frac{v}{1+t v}, \frac{(1-a t)(a+v)}{1+t v}\right) .
$$

It is easy to see that $\Psi_{a}\left(R_{a}\right)$ is a quadrangle with vertices

$$
(0,0),\left(\frac{a}{a+1}, 0\right),\left(\frac{a+1}{a+2}, \frac{a+1}{a+2}\right) \text { and }\left(0, \frac{a}{a+1}\right),
$$

from which it follows that

$$
\theta_{n+1}-\theta_{n-1} \in\left[-\frac{a_{n+1}}{a_{n+1}+1}, \frac{a_{n+1}}{a_{n+1}+1}\right] .
$$

We call the quadrangle from (7) $Q_{a}$.

Denote the Jacobean of $\Psi_{a}$ by $J_{a}$. Then

$$
\left|\operatorname{det} J_{a}\right|=\frac{a(a+2 v-a t v)}{(1+t v)^{3}} .
$$

The sequence $\Psi_{a}\left(t_{n}, v_{n}\right)=\left(\theta_{n-1}, \theta_{n+1}\right)$ is distributed over $Q_{a}$ according to the density function

$$
\frac{1}{\log 2} \frac{1}{(1+t v)^{2}} \frac{1}{\left|\operatorname{det} J_{a}\right|}
$$

which in terms of $\alpha$ and $\beta$ can be written as

$$
\frac{1}{\log 2} \frac{1}{a \sqrt{a^{2}+4 \alpha \beta}} .
$$

We call this expression $m_{a}(\alpha, \beta)$ and the measure connected with it $m_{a}$. Note the symmetry in $\alpha$ and $\beta$ of $Q_{a}$ and of $m_{a}$. This shows that the density function of the sequence $\theta_{n+1}-\theta_{n-1}, n=1,2, \ldots$, under the condition $a_{n+1}=a$, is for all $a$ and for almost all $x$ an even function. Summing all these even functions over $a$ gives an even density function on $[-1,1]$ for the sequence from (5).

In the next section we will determine this function.

\section{The Distribution of $\theta_{n+1}-\theta_{n-1}$}

Let $0 \leq z \leq \frac{a}{a+1}$. Denote by $Q_{a, z}$ that part of $Q_{a}$ that lies to the right of the line $\beta=\alpha-z$. In order to determine the distribution of the sequence $\left|\theta_{n+1}-\theta_{n-1}\right|, n=1,2, \ldots$, we calculate

$$
m_{a}\left(Q_{a, z}\right)=\frac{1}{\log 2} \iint_{Q_{a, z}} \frac{\mathrm{d} \alpha \mathrm{d} \beta}{a \sqrt{a^{2}+4 \alpha \beta}} .
$$

First we integrate over $\alpha$ :

$$
\int_{\beta+z}^{\frac{\beta}{(a+1)^{2}}+\frac{a}{a+1}} \frac{\mathrm{d} \alpha}{a \sqrt{a^{2}+4 \alpha \beta}}=\frac{1}{2 \beta}+\frac{1}{a(a+1)}-\frac{1}{2 a \beta} \sqrt{a^{2}+4 \beta^{2}+4 \beta z}
$$

Then we integrate over $\beta$, from $\beta=0$ to $\beta=\frac{a+1}{a+2}-z \frac{(a+1)^{2}}{a(a+2)}$, the last expression being the $\beta$-coordinate of the intersection point of the lines $\beta=\alpha-z$ and $\beta=$ 
$(a+1)^{2} \alpha-a(a+1)$. For this we need 1

$$
\begin{gathered}
\frac{1}{2 a} \int \frac{\sqrt{a^{2}+4 \beta^{2}+4 \beta z}}{\beta} \mathrm{d} \beta=\frac{1}{2} \log \beta+\frac{1}{2 a} \sqrt{a^{2}+4 \beta^{2}+4 \beta z} \\
+\frac{z}{2 a} \log \left(z+2 \beta+\sqrt{a^{2}+4 \beta^{2}+4 \beta z}\right)-\frac{1}{2} \log \left(a^{2}+2 \beta z+a \sqrt{a^{2}+4 \beta^{2}+4 \beta z}\right) .
\end{gathered}
$$

When substituting $\beta=\frac{a+1}{a+2}-z \frac{(a+1)^{2}}{a(a+2)}$ in the expression under the root sign, one gets at first sight a rather unpleasant result. But fortunately it is a nice square, namely

$$
\left(a+\frac{2}{a+2}-z \frac{2(a+1)}{a(a+2)}\right)^{2}
$$

and after some calculations one then finds that

$$
\begin{gathered}
m_{a}\left(Q_{a, z}\right)=\frac{1}{\log 2} \iint_{Q_{a, z}} \frac{\mathrm{d} \alpha \mathrm{d} \beta}{a \sqrt{a^{2}+4 \alpha \beta}} \\
=\frac{1}{\log 2}\left(\frac{1}{2} \log \frac{(a+1)^{2}}{a(a+2)}+\frac{z}{2 a} \log \frac{a}{a+2}+\frac{z}{2 a} \log \frac{a+z}{a-z}+\frac{1}{2} \log \left(1-\frac{z^{2}}{a^{2}}\right)\right) .
\end{gathered}
$$

For $z=0$ this is Lévy's classical expression for the frequency of a specific partial quotient:

$$
m_{a}\left(Q_{a}\right)=\frac{1}{\log 2} \log \frac{(a+1)^{2}}{a(a+2)} ;
$$

see e.g. [1], page 85. Thus we have found the following result:

Theorem 2. For almost all $x$ the distribution function on the interval $\left[0, \frac{a}{a+1}\right]$ of the sequence

$$
\left|\theta_{n+1}-\theta_{n-1}\right|, n=1,2, \ldots
$$

under the condition $a_{n+1}=a$ is

$$
\frac{1}{\log 2}\left(\frac{z}{a} \log \frac{a+2}{a}+\frac{z}{a} \log \frac{a-z}{a+z}-\log \left(1-\frac{z^{2}}{a^{2}}\right)\right),
$$

and the corresponding density function

$$
\frac{1}{\log 2}\left(\frac{1}{a} \log \frac{a+2}{a}+\frac{1}{a} \log \frac{a-z}{a+z}\right)
$$

We call these functions $D_{a}$ and $d_{a}$, respectively, and extend their definitions by

$$
D_{a}(z)=0 \text { for } z<0, \quad D_{a}(z)=\frac{1}{\log 2} \log \frac{(a+1)^{2}}{a(a+2)} \text { for } z>\frac{a}{a+1}
$$

and

$$
d_{a}(z)=0 \quad \text { outside } \quad\left[0, \frac{a}{a+1}\right] .
$$

Observe that $d_{a}$ has only one point of discontinuity, namely $z=0$.

The functions $D$ and $d$, defined by

$$
D(z)=\sum_{a=1}^{\infty} D_{a}(z) \text { and } d(z)=\sum_{a=1}^{\infty} d_{a}(z)
$$

\footnotetext{
${ }^{1}$ Found with the help of Wolfram Mathematica Online Integrator and verified by differentiation.
} 
are the distribution and the density function of the sequence $\left|\theta_{n+1}-\theta_{n-1}\right|$ on the interval $[0,1]$. The function $d$ is monotonically decreasing. Its value in 0 is

$$
\frac{1}{\log 2} \sum_{a=1}^{\infty} \frac{1}{a} \log \left(1+\frac{2}{a}\right)=3.05615 \cdots
$$

and

$$
d^{\prime}(z)=-\frac{\pi^{2}}{3 \log 2}+O(z), \quad z \downarrow 0
$$

4. The mean distance Between $\theta_{n-1}$ And $\theta_{n+1}$

We now determine, for almost all $x$, the mean distance between $\theta_{n-1}$ and $\theta_{n+1}$. This means that we first have to calculate

$$
\int_{0}^{\frac{a}{a+1}} z d_{a}(z) \mathrm{d} z
$$

which is easy, using

$$
\int z \log \frac{a-z}{a+z} \mathrm{~d} z=-a z-\frac{1}{2}\left(a^{2}-z^{2}\right) \log \frac{a-z}{a+z} .
$$

It gives

and so

$$
\int_{0}^{\frac{a}{a+1}} z d_{a}(z) \mathrm{d} z=\frac{1}{\log 2}\left(-\frac{a}{a+1}+\frac{1}{2} a \log \frac{a+2}{a}\right),
$$

$$
\int_{0}^{1} z d(z) \mathrm{d} z=\frac{1}{\log 2} \sum_{a=1}^{\infty}\left(-\frac{a}{a+1}+\frac{1}{2} a \log \frac{a+2}{a}\right) .
$$

Now

$$
\sum_{a=1}^{n} \frac{a}{a+1}=n-\log n+1-\gamma+O\left(\frac{1}{n}\right), \quad n \rightarrow \infty
$$

and

Trivially,

$$
\sum_{a=1}^{n} a \log \frac{a+2}{a}=(n-1) \log (n+1)+n \log (n+2)-2 \sum_{a=1}^{n} \log a .
$$

$$
(n-1) \log (n+1)+n \log (n+2)=2 n \log n-\log n+3+O\left(\frac{1}{n}\right), \quad n \rightarrow \infty,
$$

and by Stirling's formula,

$$
\sum_{a=1}^{n} \log a=n \log n-n+\frac{1}{2} \log n+\frac{1}{2} \log 2 \pi+O\left(\frac{1}{n}\right), n \rightarrow \infty .
$$

The final outcome is that

$$
\int_{0}^{1} z d(z) \mathrm{d} z=\frac{1}{\log 2}\left(\gamma+\frac{1}{2}-\frac{1}{2} \log 2 \pi\right) .
$$

This finishes the proof of the following theorem:

Theorem 3. For almost all $x$,

$$
\lim _{N \rightarrow \infty} \frac{1}{N} \sum_{n=1}^{N}\left|\theta_{n+1}-\theta_{n-1}\right|=\frac{2 \gamma+1-\log 2 \pi}{2 \log 2}=0.22834 \ldots,
$$

where $\gamma$ is Euler's constant. 


\section{REFERENCES}

[1] Karma Dajani and Cor Kraaikamp, Ergodic Theory of Numbers, The Mathematical Association of America, The Carus Mathematical Monographs 29, (2002). MR1917322 (2003f:37014)

[2] J. F. Koksma, Diophantische Approximationen, Verlag Julius Springer, Berlin 1937. MR 0344200 (49:8940)

[3] H. Jager, The distribution of certain sequences connected with the continued fraction, Indag. Math. Series A 89, 1986, 61-69. MR834320 (87g:11092)

Oude Larenseweg 26, 7214 PC Epse, the Netherlands

E-mail address: epserbos@xs4all.nl 